\title{
Tunka-133: Results of 3 year operation ${ }^{\text {is }}$
}

\author{
V.V. Prosin ${ }^{\mathrm{a}, *}$, S.F. Berezhnev ${ }^{a}$, N.M. Budnev ${ }^{\mathrm{b}}$, A. Chiavassa $^{\mathrm{e}}$, O.A. Chvalaev ${ }^{\mathrm{b}}$, O.A. Gress ${ }^{\mathrm{b}}$, \\ A.N. Dyachok ${ }^{\text {b }}$, S.N. Epimakhov ${ }^{g}$, N.I. Karpov ${ }^{\text {a }}$, N.N. Kalmykov ${ }^{\text {a }}$, E.N. Konstantinov ${ }^{\text {b }}$, \\ A.V. Korobchenko ${ }^{\text {, }}$, E.E. Korosteleva ${ }^{\text {a }}$, V.A. Kozhin ${ }^{\text {a }}$, L.A. Kuzmichev ${ }^{\text {a }}$, \\ B.K. Lubsandorzhiev ${ }^{c}$, N.B. Lubsandorzhiev ${ }^{c}$, R.R. Mirgazov ${ }^{b}$, M.I. Panasyuk ${ }^{\text {a }}$, L.V. Pan'kov ${ }^{b}$, \\ E.G. Popova ${ }^{a}$, V.S. Ptuskin ${ }^{d}$, Yu.A. Semeney ${ }^{b}$, A.A. Silaev ${ }^{\text {a }}$, A.A. Silaev Jr. ${ }^{a}$, A.V. Skurikhin ${ }^{\text {, }}$, \\ C. Spiering ${ }^{f}$, L.G. Sveshnikova ${ }^{a}$, I.V. Yashin ${ }^{a}$, A.V. Zagorodnikov ${ }^{b}$
}

a Skobeltsyn Institute of Nuclear Physics MSU, Moscow, Russia

${ }^{\mathrm{b}}$ Institute of Applied Physics ISU, Irkutsk, Russia

${ }^{\mathrm{c}}$ Institute for Nuclear Research of RAS, Moscow, Russia

d IZMIRAN, Troitsk, Moscow Region, Russia

e Dipartimento di Fisica Generale Università di Torino and INFN, Torino, Italy

${ }^{\mathrm{f}}$ DESY, Zeuthen, Germany

${ }^{\mathrm{g}}$ Hamburg University, Hamburg, Germany

\section{A R T I C L E I N F O}

Available online 17 September 2013

Keywords:

Tunka-133

Spectrum

Composition

Cherenkov light

\begin{abstract}
A B S T R A C T
The EAS Cherenkov light array Tunka-133, with $\sim 3 \mathrm{~km}^{2}$ geometric area, is taking data since 2009. The array permits a detailed study of cosmic ray energy spectrum and mass composition in the PeV energy range. After a short description of the methods of EAS parameter reconstruction, we present the allparticle energy spectrum and results of studying CR composition, based on 3 seasons of array operation. In the last part of the paper, we discuss possible interpretations of the obtained results.
\end{abstract}

(c) 2013 Published by Elsevier B.V.

\section{Introduction}

The study of primary energy spectrum and mass composition in the energy range of $10^{15}-10^{18} \mathrm{eV}$ is of crucial importance for understanding origin and propagation of cosmic rays (CR) in the Galaxy. An increasing dominance of heavy nuclei above the "knee" up to $10^{17} \mathrm{eV}$ indicated the energy limit of $\mathrm{CR}$ acceleration in galactic sources. At higher energies, the composition becomes lighter again up to $2 \times 10^{18}$. This may indicate a transition to an extragalactic origin of CR.

To measure the primary energy spectrum and mass composition of cosmic rays in the mentioned energy range, the array Tunka-133 [1,2] with nearly $3 \mathrm{~km}^{2}$ geometrical area has been deployed in the Tunka Valley, Siberia. It records EAS Cherenkov light using the atmosphere of the Earth as a huge calorimeter, resulting in a better energy resolution $(\sim 15 \%)$ than EAS arrays detecting only charged particles. The detectors are grouped into 25 clusters, each with 7 detectors - six hexagonally arranged detectors and one in the center. The distance between the detectors in

\footnotetext{
This article is part of the special issue ' 4 th Roma International Conference on Astroparticle Physics'. The other articles of this special issue were published in the issue 742 .

* Corresponding author. Tel.: +7 49593910 72; fax: +7 4959393553.

E-mail address: v-prosin@yandex.ru (V.V. Prosin).
}

the cluster is $85 \mathrm{~m}$. 19 clusters are installed in an inner circle of $500 \mathrm{~m}$ radius - "inside" clusters, 6 clusters were placed at the distance of 700 - $1000 \mathrm{~m}$ from the center - "outside" clusters. Tunka-133 operates in clear moonless nights every year from October up to the beginning of April. Results presented in this paper are based on the data of the first three seasons of the array operation. The total time of data acquisition is $980 \mathrm{~h}$. The number of recorded events is about $6 \times 10^{6}$.

\section{EAS parameter reconstruction}

The primary data record for each Cherenkov light detector contains 1024 amplitude values in steps of $5 \mathrm{~ns}$ [1]. Thus each pulse waveform is recorded over $5 \mu$ s. To derive the three main parameters of the pulse: pulse peak amplitude $A_{i}$, front delay $t_{i}$ at a level 0.25 of $A_{i}$, and pulse area $Q_{i}$, each pulse is fitted with a specially developed smoothing curve [3]. A fourth pulse parameter is the effective width $\tau_{\text {eff }}$ equal $Q_{i} /\left(1.24 \cdot A_{i}\right)$. The accuracy of this parameter is better than that of the pulse width (FWHM) used earlier. The additional coefficient (1.24) "normalizes" $\tau_{\text {eff }}$ to FWHM.

The reconstruction of the EAS core position is performed by fitting measured amplitudes $A_{i}$ with an amplitude distance 
function (ADF)

$A(R)=A(200) \cdot f(R)$,

The function $f(R)$ is a fit to four different parameterizations according to the distance $R$ (in meters) to the shower core

$$
f(R)= \begin{cases}\exp \left(\frac{\left(R_{\mathrm{kn}}-R\right)}{R_{0}}\left(1+\frac{3}{R+2}\right)\right), & R<R_{\mathrm{kn}} \\ \left(\frac{200}{R}\right)^{b_{2}}, & 200 \mathrm{~m} \geq R \geq R_{\mathrm{kn}} \\ \left(\left(\frac{R}{200}+a\right) /(1+a)\right)^{-b_{A}}, & 400 \mathrm{~m} \geq R \geq 200 \mathrm{~m} \\ \left(\left(\frac{R}{200}+1\right) / 2\right)^{-b_{A}}, & R>400 \mathrm{~m}\end{cases}
$$

All four variables in Eq. (3) $\left(R_{0}, R_{\mathrm{kn}}, a\right.$ and $\left.\mathrm{b}_{2}\right)$, describing the ADF shape in the different ranges of core distance, are related to a single parameter of the ADF shape - the steepness $b_{A}$

$d=b_{A}-5, \quad \lg d=\log d$

$R_{0}=275 / d$

$R_{\mathrm{kn}}=145-115 \cdot \lg d$

$a=0.89-0.29 \cdot \lg d$

$b_{2}= \begin{cases}2.4+2 \cdot(\lg d-0.15), & b_{A} \geq 6.41 \\ 2.4, & b_{A}<6.41\end{cases}$

As a measure of energy we use the Cherenkov light flux density at a core distance of $200 \mathrm{~m}-\mathrm{Q}(200)$. The connection between the EAS energy $E_{0}$ and $Q(200)$ can be expressed by the following formula:

$E_{0}=C \cdot Q(200)^{g}$.

It was found from CORSIKA simulations that for the energy range of $10^{16}-10^{18} \mathrm{eV}$, the zenith angle range of $0-45^{\circ}$ and a complex composition, consisting of equal contribution of protons and iron nuclei, the value of the index $g$ is 0.94 .

To reconstruct the EAS energy from the Cherenkov light flux one needs to know the absolute sensitivities of the Cherenkov detectors and the atmosphere transparency. To avoid these problems, the method of normalization of the integral energy spectrum to a reference spectrum is used. The reference energy spectrum was measured by the QUEST experiment [4,5]. The integral energy spectrum obtained for each night of the Tunka133 operation is normalised to that reference spectrum.

\section{Energy spectrum}

For the reconstruction of the energy spectrum, events with a core position inside a circle of radius $R=450 \mathrm{~m}$ from the center of the array were selected. The EAS zenith angle of events used for the spectral measurement was constrained to $<45^{\circ}$. With these selections, a $100 \%$ registration efficiency is reached for energies larger than $6 \times 10^{15} \mathrm{eV}$. The total statistics above that energy is 170000 events, 60000 of them with $E_{0} \geq 10^{16} \mathrm{eV}$ and 600 with $E_{0} \geq 10^{17} \mathrm{eV}$.

We found that also for events with $R$ between $450 \mathrm{~m}$ and $800 \mathrm{~m}$, the energy spectrum can be reconstructed. Above some energy threshold $\left(5 \times 10^{16} \mathrm{eV}\right)$ it is in good agreement with the spectrum for events with $R<450 \mathrm{~m}$ [1]. Based on these results we reconstruct the combined energy spectrum (Fig. 1) for events with $R<450 \mathrm{~m}$ for $E_{0}<10^{17} \mathrm{eV}$ and events with $R<800 \mathrm{~m}$ for higher energies. The combined spectrum contains about 1900 events with $E_{0}>10^{17} \mathrm{eV}$.

The energy spectrum of Tunka-133 is compared with that of Tunka-25 [6], the predecessor of Tunka-133, in Fig. 1. The energy spectrum above the knee looks rather complicated. One can see that the spectrum can be fitted by power laws with 3 different power law indices: $3.23 \pm(0.01)^{\text {stat }} \pm(0.05)^{\text {syst }}$ for $6 \times 10^{15}-2$

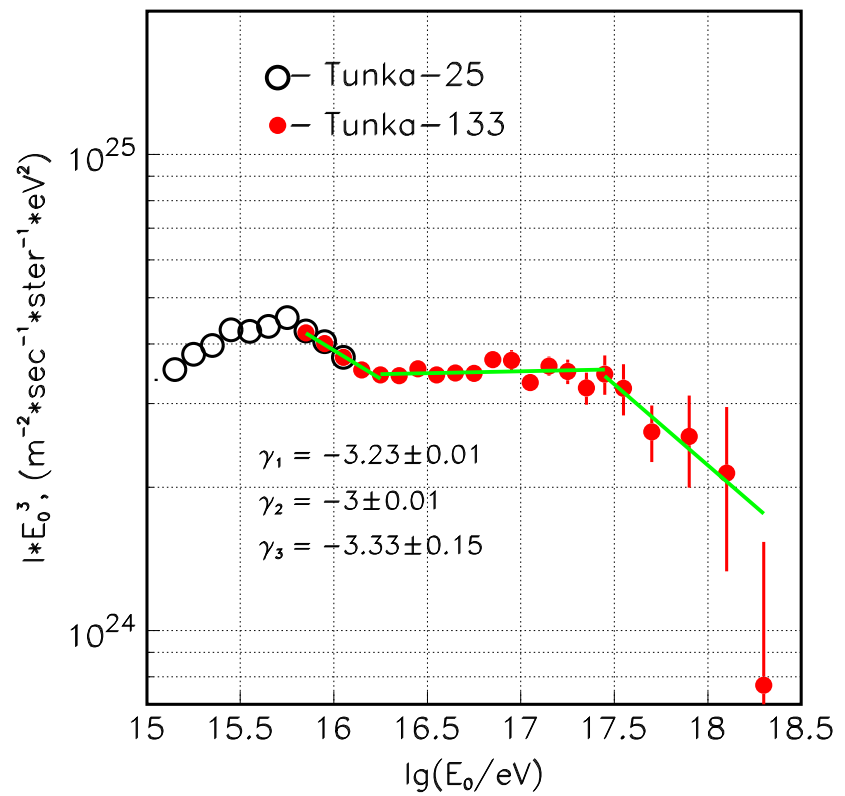

Fig. 1. Primary CR energy spectrum. (For interpretation of the references to colour in this figure caption, the reader is referred to the web version of this paper.)

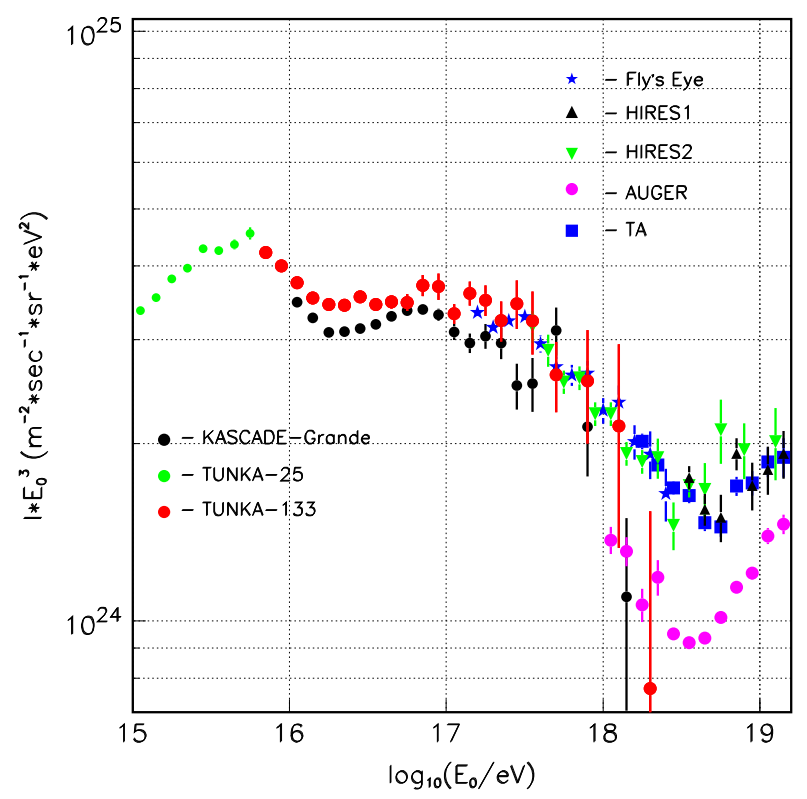

Fig. 2. Combined primary energy spectrum: comparison with some other experimental data.

$\times 10^{16} \mathrm{eV}, \quad 3.00 \pm(0.01)^{\text {stat }} \pm(0.05)^{\text {syst }}$ for $2 \times 10^{16}-3 \times 10^{17} \mathrm{eV}$, $3.33 \pm(0.15)^{\text {stat }} \pm(0.05)^{\text {syst }}$ for $3 \times 10^{17}-10^{18} \mathrm{eV}$.

A comparison of the Tunka-133 spectrum with the results of other experiments is presented in Fig. 2. The energy range covered by spectrum presented here $\left(10^{16}-10^{18} \mathrm{eV}\right)$ is nearly the same as covered by the KASCADE-Grande array data [7].

Both spectra reproduce the same structures: a decrease of the power law index at $2 \times 10^{16} \mathrm{eV}$ and an increase at $3 \times 10^{17} \mathrm{eV}$.

The points of the Tunka-133 spectrum coincide with that of the Fly's Eye and HiRes experiments in the range of $2 \times 10^{17}-10^{18} \mathrm{eV}$. These spectra are consistent with the spectrum of the present TA array at higher energies. 


\section{Mass composition}

To get a uniform estimation for $b_{A}$ over a wide range of energies, we remove from the analysis detectors at core distances larger than $250 \mathrm{~m}$ during the last step of parameter reconstruction.

\subsection{Two methods of $X_{\max }$ measurement}

Recording the pulse waveform for each detector allows to use two methods of $X_{\max }$ reconstruction, which were developed for our experiment. The first is based on the shape of the ADF (see above) and called $b$-method. The second method, the $\tau$-method, is based on an analysis of the width of the Cherenkov pulses.

The ADF shape is described by an expression with a single parameter, the steepness $b_{A}$ (see above). CORSIKA simulation proves that the steepness $b_{A}$ is strictly connected with the relative position of the EAS maximum $\left(\Delta X_{\max }=X_{0} / \cos \theta-X_{\max }\right)$

$\Delta X_{\max }=A-B \cdot \log \left(b_{A}-2\right)$

MC simulations show that this relation does not depend on energy, zenith angle of showers, mass composition and the model of nuclear interaction used for the simulation. A plot of 503 simulated events both for protons and iron, for the energy range $10^{16}-10^{17} \mathrm{eV}$ and zenith angles from $0^{\circ}$ to $45^{\circ}$, is shown in Fig. 3.

The $\tau$-method uses the sensitivity of the pulse width at some fixed core distance to the position of the EAS maximum. We fixed this distance to $400 \mathrm{~m}$ and recalculated the values measured with detectors at distances between 200 and $450 \mathrm{~m}$ from the core. To recalculate the pulse width to $400 \mathrm{~m}$, the width-distance function (WDF) is used. This function was constructed on the basis of CORSIKA simulation and described in Ref. [2]. It was also shown [2] that the value of $\tau(400)$ is connected with the thickness of the atmosphere between the detector and $X_{\max }\left(\Delta X_{\max }=X_{0} / \cos \theta-X_{\max }\right)$ by the expression

$\Delta X_{\max }=C-D \cdot \log \tau_{\text {eff }}(400)$

This relation is correct for any primary nucleus, any energy and zenith angle of the shower and any interaction model, as in the case of ADF steepness mentioned above. The plot of the simulated events is shown in Fig. 4.

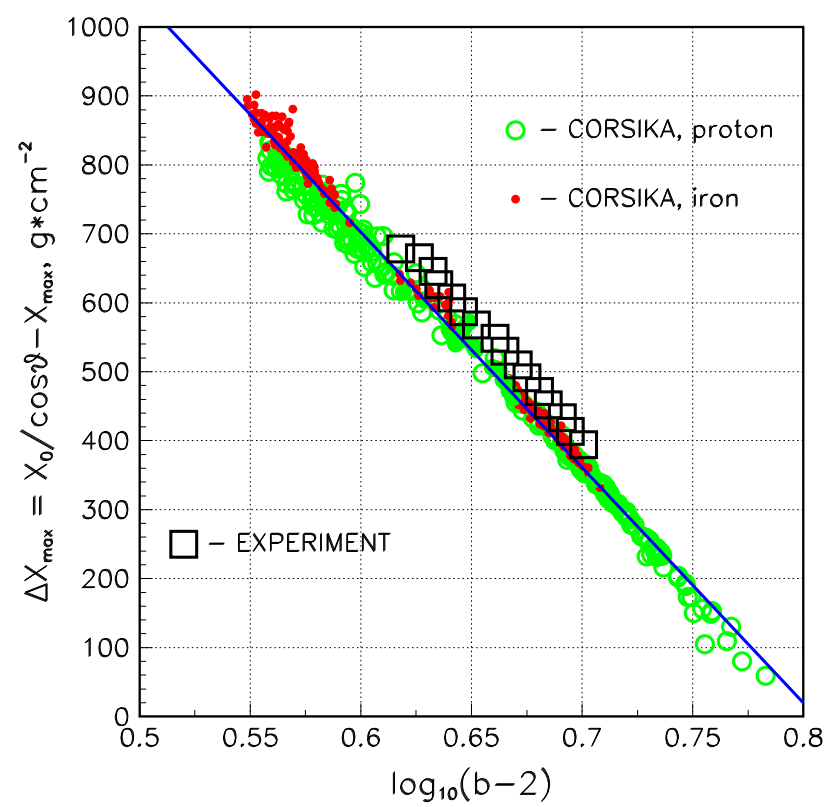

Fig. 3. Dependence of the relative EAS maximum position $\Delta X_{\max }$ on ADF steepness $\log \left(b_{A}-2\right)$.

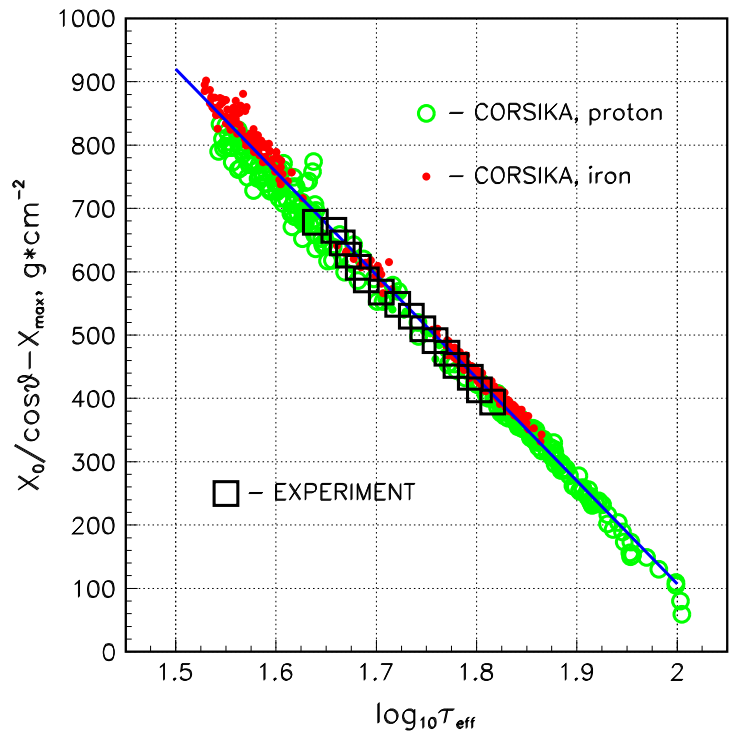

Fig. 4. Dependence of the relative EAS maximum position $\Delta X_{\max }$ on $\log \tau_{\text {eff }}(400)$.

\subsection{Phenomenological approach}

The phenomenological approach means the experimental check of the correlation between the measured parameter of the shower and the position of the maximum. We start from the parameter $\tau_{\text {eff }}(400)$. Its zenith angle dependence (log $\tau_{\text {eff }}(400)$ vs. $1 / \cos \theta)$ can be easily recalculated to the connection between $\log \left(\tau_{\text {eff }}(400)\right)$ and $\Delta X_{\max }$. The only value which we need to add to this analysis is the mean $\left\langle X_{\max }\right\rangle$ for the energy bin chosen for the analysis. The logarithmic energy bin $16.4<\log \left(E_{0} / \mathrm{eV}\right)<16.5$ is chosen, and it is assumed that $\left\langle X_{\max }\right\rangle=580 \mathrm{~g} \mathrm{~cm}^{-2}$ for this energy. As a result we get consistency with the CORSIKA simulated correlation as shown in Fig. 4. The experimental points are obtained from about 3600 events in this energy bin. The phenomenological values of the constants in expression (6) are as follows: $C=3344 \mathrm{~g} \mathrm{~cm}^{-2}, D=1624 \mathrm{~g} \mathrm{~cm}^{-2}$.

A similar procedure for the same energy has been used for the second parameter $b_{A}$. The result is shown in Fig. 3. The experimental dependence deviates slightly from the simulated one. This can happen because of the more complicated character of the parameter $b_{A}$. The phenomenological points can be fitted with expression (5) with the values: $A=2865 \mathrm{~g} \mathrm{~cm}^{-2}, B=3519 \mathrm{~g} \mathrm{~cm}^{-2}$.

These phenomenological expressions have been used for the measurement of $X_{\max }$ for each individual event.

\subsection{Estimation of $X_{\max }$ experimental error}

The use of two independent methods of $X_{\max }$ measurement for each individual event allows estimating the experimental error $\sigma_{\text {exp }}\left(X_{\max }\right)$ using the experimental data itself. We analyze the distribution of the difference: $\delta X_{\max }=X_{\max , \tau}-X_{\max , b}$ - for each energy bin. The RMS of the $\delta X_{\max }$ distribution changes slightly from $48 \mathrm{~g} \mathrm{~cm}^{-2}$ at $E_{0}=10^{16} \mathrm{eV}$ to $40 \pm 1 \mathrm{~g} \mathrm{~cm}^{-2}$ for energies $E_{0} \geq$ $3 \times 10^{16} \mathrm{eV}$. Assuming equal experimental errors for any of the methods we can estimate $\sigma_{\text {exp }}\left(X_{\max }\right)=\delta X_{\max } / \sqrt{2}$. So $\sigma_{\exp }=28 \pm$ $1 \mathrm{~g} \mathrm{~cm}^{-2}$ for $E_{0} \geq 3 \times 10^{16} \mathrm{eV}$. This value is used in paragraph 5 for the analysis of experimental $X_{\max }$ distributions (see also Ref. [8]) .

\section{4. $X_{\max }$ vs. $E_{0}$}

The experimental dependence of mean $\left\langle X_{\max }\right\rangle$ vs. primary energy $E_{0}$ in the energy range of $6 \times 10^{15}-5 \times 10^{17} \mathrm{eV}$ obtained with the two methods described above is presented in Fig. 5. Only 


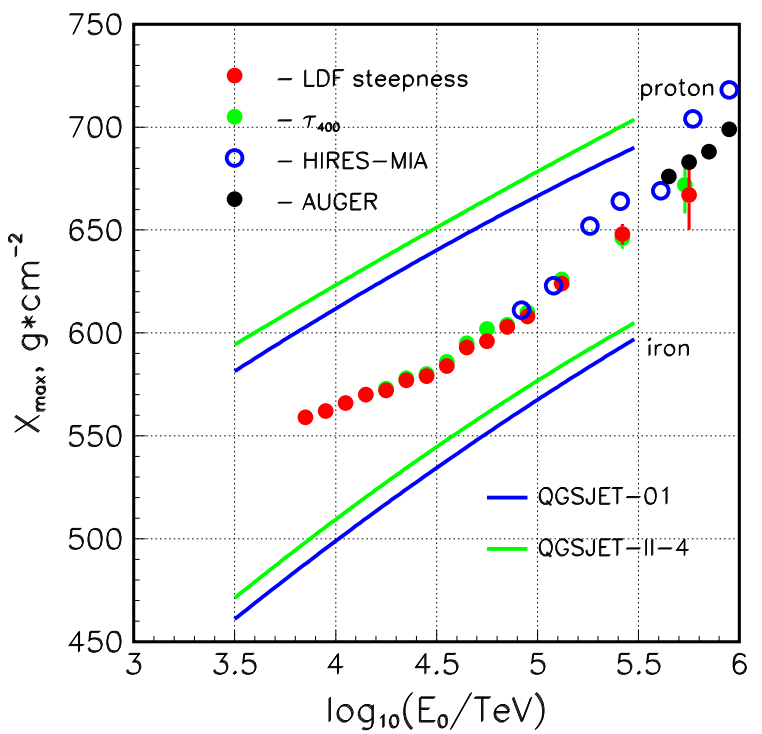

Fig. 5. Experimental $X_{\max }$ vs. primary energy.

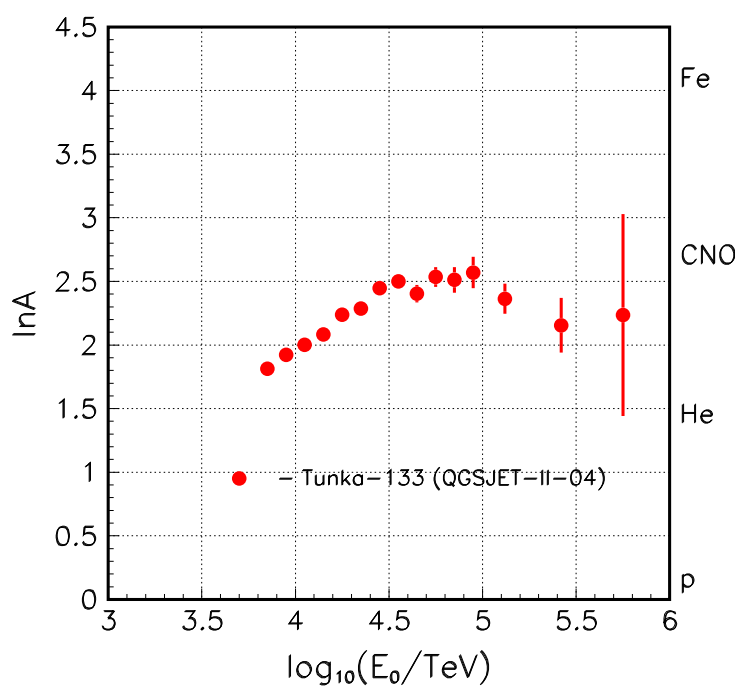

Fig. 6. Experimental $\langle\ln A\rangle$ vs. primary energy.

events where the difference between the two $X_{\max }$ values is less than $3 \delta X_{\max }$ were used. The new measurements are compared with the theoretical curves simulated with the old QGSJET-01 and the very new QGSJET-II-04 model for primary protons and iron nuclei. It is seen from Fig. 5 that the new model provides a depth of the EAS maximum about $10 \mathrm{~g} \times \mathrm{cm}^{-2}$ higher than the old one.

The mean values of $\left\langle X_{\max }\right\rangle$ can be recalculated to the mean values of $\langle\ln A\rangle$ by a simple method of interpolation. The result of such an approach for the points derived from the ADF steepness analysis is shown in Fig. 6.

\section{Elemental composition spectrum}

Analysis of $X_{\max }$ distribution gives the possibility of reconstruction energy spectrum of different nucleus groups. In our case we use 4 groups: p, He, CNO and Fe.

For this analysis, partial $X_{\max }$ distributions were simulated using CORSIKA 7.35 (2013) with an updated version of the high-energy interaction model QGSJETII-04/GHEISHA, based on recent LHC data at $\sqrt{s}=7 \mathrm{TeV}$ [9]. Sets of simulated events were produced for $5 \log$-equidistant energies from $10^{15.5}$ to $10^{17.5} \mathrm{eV}$ and for 4 different representative mass groups (proton, helium, nitrogen, iron) with 10000 vertical showers per energy and group using statistical thinning $\varepsilon=10^{-4}$ with the weight $w=\varepsilon E(\mathrm{GeV})$. Thus, the total number of simulated showers is 200000 .

Each partial distribution is fitted by a shifted Gamma distribution with the following probability density function:

$P_{G}\left(X_{\max }\right)=\frac{\left(X_{\max }-X_{0}\right)^{\gamma-1}}{\Gamma(\gamma) \beta^{\gamma}} \exp \left(-\frac{X_{\max }-X_{0}}{\beta}\right)$

for $X_{\max } \geq X_{0} ; \gamma, \beta>0$.

Mean and standard deviation

$\left\langle X_{\max }\right\rangle=\beta \gamma+X_{0}$

$R M S=\beta \sqrt{\gamma}$.

Statistical uncertainties of $\left\langle X_{\max }\right\rangle$ and RMS are less than $1 \mathrm{~g} \mathrm{~cm}^{2}$.

To recalculate distributions of nuclei for all energy intervals interpolations of Gamma distribution parameters are used. For interpolation physically sensible parameters $\left\langle X_{\max }\right\rangle$, RMS and $\gamma$ index were chosen [8]. Each gamma distribution is convoluted with Gaussian with known $X_{\max }$ resolution and used for fitting.

The composition of $\mathrm{CR}$ is determined by the fit procedure of the EAS depth distributions as a superposition of weighted elemental distributions in narrow logarithmic intervals of 0.1 of the reconstructed primary energy from $7 \times 10^{15}$ to $10^{17} \mathrm{eV}$ and in three merged intervals above $10^{17} \mathrm{eV}$.

The weights of each group are found through log-likelihood minimization and can be used for recalculation of elemental spectra and mean natural logarithm of mass $A$.

However, due to the fact that the fit function has a quite irregular behavior for minimization it is difficult to find a reliable minimum after a certain number of iterations. Procedure requests to specify initial values and the result of fitting depends on these values and statistical uncertainties of experimental distributions. As initial values, weights are ranged with step of $10 \%$. Furthermore, to suppress the influence of statistical fluctuations on the result each bin of the experimental distribution is varied according to Poissonian law with a mean equal to the experimental value. Mean and standard deviation of found solutions are accepted as resultant composition and error of the method. The best fit for three different energy bins is shown in Fig. 7.

Fig. 8 represents "light" $(\mathrm{H}+\mathrm{He})$ and "heavy" fraction $(\mathrm{N}+\mathrm{Fe})$ in comparison with recent results of KASCADE-Grande [11].

The results for the elemental spectra of proton, helium, nitrogen and iron of the $X_{\max }$ based analysis are shown in Fig. 9.

Features of the all-particle spectrum can be explained from the standpoint of the mass composition. Our investigations reveal that the complex "knee" at $3-6 \times 10^{15} \mathrm{eV}$ in the all-particle spectrum is associated with a limit of acceleration of the proton and helium nuclei in the Galaxy. Moreover, from the energy $3 \times 10^{16} \mathrm{eV}$ and higher a similar growth of the light component caused by a potential extragalactic modulation can be seen (see next paragraph). The same "knee-like" structure in the spectrum of the heavy component is obtained at $7 \times 10^{16} \mathrm{eV}$. Both components have equivalent fractions at energies of $10^{16}$ and $3-5 \times 10^{17} \mathrm{eV}$.

This behaviour implies an increase of the mean logarithmic of mass $\langle\ln A\rangle$ as a function of energy (Fig. 10) up to $10^{17} \mathrm{eV}$ from 2.0 to 2.7. At energies higher than $10^{17} \mathrm{eV}$ the composition is becoming lighter with $\langle\ln A\rangle$ going down to 2.1 at an energy of $6 \times 10^{17} \mathrm{eV}$. The obtained $\langle\ln A\rangle$ has a quite smooth behaviour and is consistent within experimental errors with KASCADE [10] and fluorescence experiments HiRES/MIA[12] and Auger[13]. In present study, the $\langle\ln A\rangle$ of KASCADE are inferred from elemental spectra of an unfolding procedure for QGSJET01c/GHESHA hypothesis. In the case of fluorescence experiments, the simple interpolation of $\left\langle X_{\max }\right\rangle$ between proton and iron is used. 

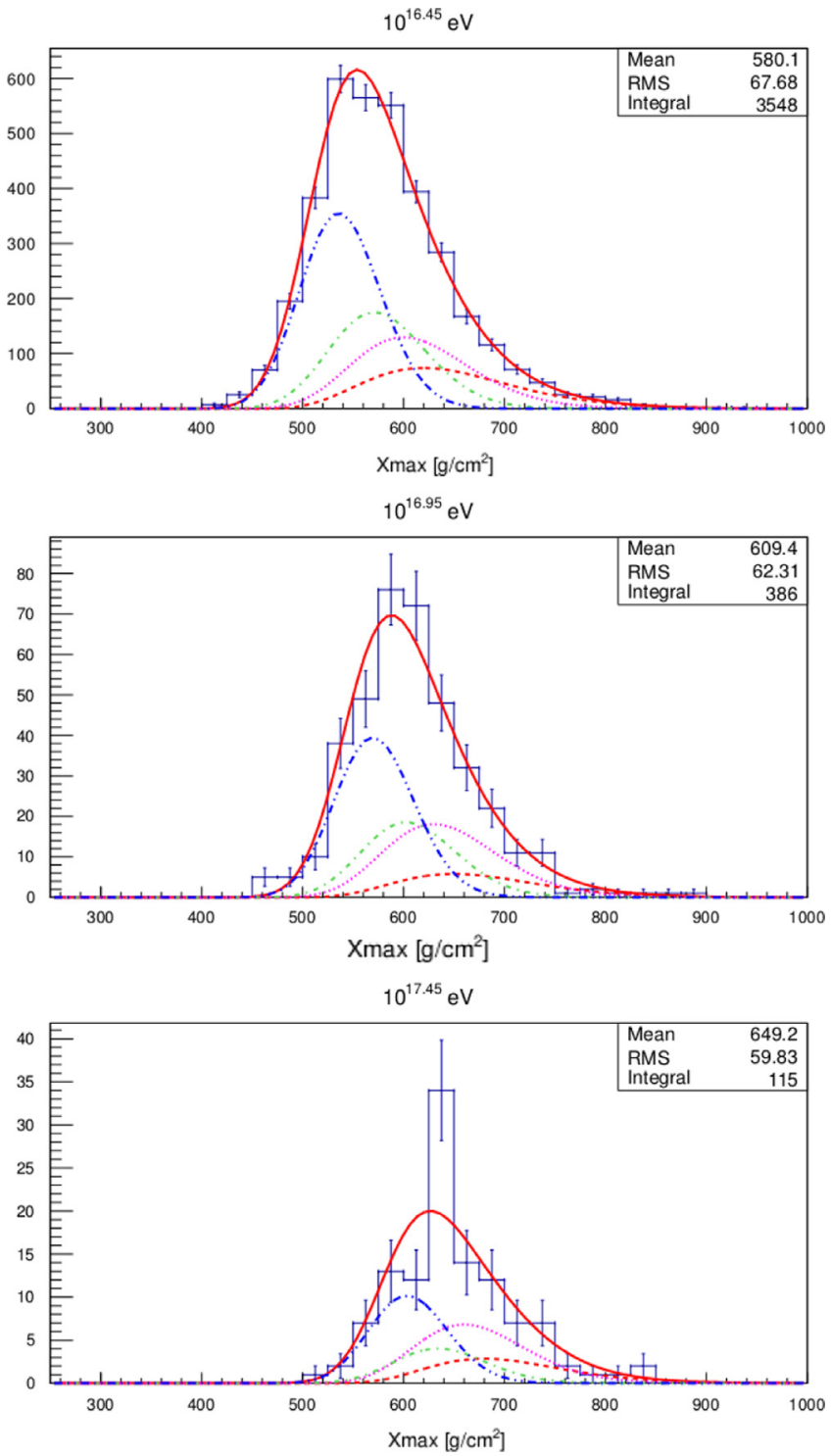

Fig. 7. Best fit (solid) for three different energy bins. The lines correspond to: proton (dash), helium (dot-dot), nitrogen (dash-dot) and iron (dash-dot-dot).

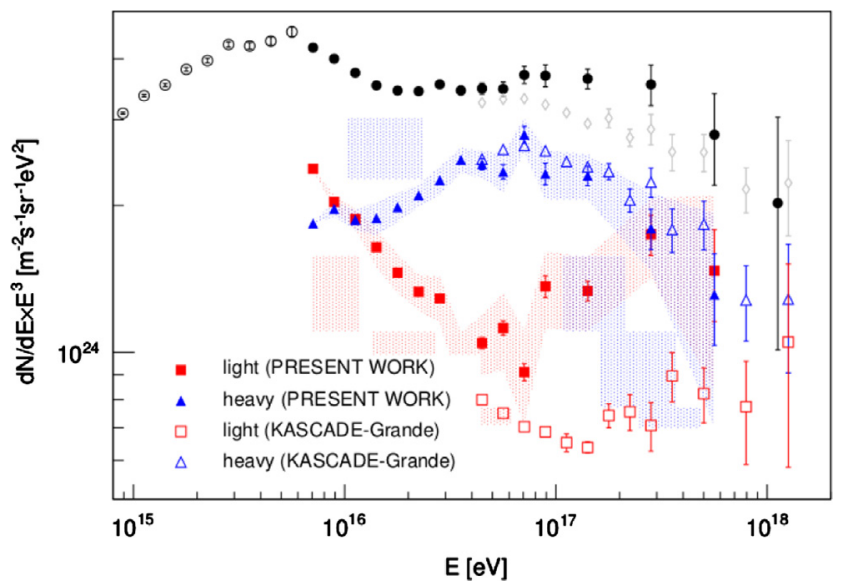

Fig. 8. Spectra of "light" and "heavy" fractions as compared with KASCADE-Grande.

Moreover, the influence of high-energy interaction models on the results inferring the mass composition has been considered. The choice of model affects mainly the absolute position and, to a lesser extent, the width of partial $X_{\max }$ distributions of nuclei. Thus, it was found that the difference between QGSJETII-04 and QGSJET01c models for proton and for iron is about $10 \mathrm{~g} \mathrm{~cm}^{2}$ for $\left\langle X_{\max }\right\rangle$ and for RMS less than $1.5 \mathrm{~g} \mathrm{~cm}^{2}$. It gives a systematic shift $\Delta(\langle\ln A\rangle)=0.2-0.3$ toward to heavier composition. This fact means that the partial $X_{\max }$ distribution has the universal shape, depending only on the primary energy and the type of nucleus.

\section{Galactic and extragalactic components of cosmic rays}

All particle energy spectrum and dependence of mean $\ln A$ on energy may be explained in the model combining energy spectrum of extragalactic protons, calculated in Refs. [17,18] with spectrum of galactic CRs [15,16].

Galactic CRs are produced by core collapse SNRs up to energy of $2 \mathrm{PeV}$ with decreasing number of SNRs accelerating up to $E_{\max }$ as $N\left(>E_{\max }\right) \sim E_{\max }^{-0.17}[14]$. Only $\sim 20 \%$ of all types of SNRs accelerate CR up to $E_{\max } \sim 4 \times Z \mathrm{PeV}$, with a small dispersion. The energy of $E=26 \times(4 \pm 1) \mathrm{PeV}$ is an upper limit of galactic CR accelerators. The source spectrum has a slope $\gamma \sim-2.2$ up to $E_{\max }$ and it is steepening by $d \gamma \sim-1.5$ above $E_{\max }$ with the parameter $\omega=4$ that provides the sharpness of the knee.

Protons with energy of $E<10^{17} \mathrm{eV}$ diffusing in extragalactic magnetic fields cannot reach the observer on the Earth within a given time due to expansion of the Universe [18] that leads to a low energy cut-off of them (this effect is called 'magnetic horizon').

We include in our calculations three fits of extragalactic protons spectrum (see thin lines 1, 2, 3 in Fig. 11) that are within the diversity of predictions obtained in Ref. [18].

In Fig. 11 we present the all particle spectrum as well as in Fig. 12 we show the corresponding energy dependence of $\langle\ln A\rangle$ calculated in frames of the model described above.

The total cosmic ray galactic component is marked by a dark green line in Fig. 11. Our study shows that a smoother cut-off in the source spectrum $(d \gamma<-1.5$ or exponential cut-off) does not allow us to describe the overall sharpness of the knee. A chemical composition of CR accelerated in the core collapse SNRs was chosen as 'normal': ( $\sim 36 \%$ of $\mathrm{H}, \sim 24 \%$ of $\mathrm{He}, 10 \%$ of $\mathrm{CNO}, 9 \%$ of $\mathrm{Fe}$ ) at the energy of $1 \mathrm{TeV}$ However we need to tune up the composition of cosmic rays accelerated in SN Ia remnants: the Fe nuclei portion was increased up to $15 \%$ to describe the spectrum of heavy nuclei around $10^{18} \mathrm{eV}$ (see the orange line for $Z>20$ in Fig. 1 and the green line for $Z>14$ ). The He portion was also increased; as a result the chemical composition of galactic $C R$ at $E \sim 1 \mathrm{PeV}$ differs from the normal one: $17 \%$ of $\mathrm{H}, 46 \%$ of $\mathrm{He}, 8 \%$ of $\mathrm{CNO}, 16 \%$ of Fe nuclei.

In Refs. $[14,16]$ the spectrum hardening at $(2-3) \times 10^{16} \mathrm{eV}$ was caused by a rise of heavy component of galactic CR. But this hypothesis requires a very sharp cut-off in the source spectrum with $d \gamma \sim 2.5$ and $\omega \sim 6$. In the present model the spectrum hardening marks a rise of extragalactic protons contribution beginning from (2-8) $10^{7} \mathrm{GeV}$. A relatively sharp suppression of the low energy part of extragalactic protons in Ref. [18] together with a sharp steepening of the galactic spectrum around the energy $\sim 4 \times 26 \mathrm{PeV}$ in our model leads to the 'second knee' structure, seen at the border between galactic and extragalactic cosmic rays (as it was predicted in Ref. [18]) in all three cases of extragalactic CR contributions (three thin lines $1,2,3)$. The main signatures of this hypothesis are a moderate contribution of subFe nuclei around $(2-3) 10^{17} \mathrm{eV}$ ( $\sim 50 \%$, not higher), and a wide $\ln A-$ distribution (a big value of $\sigma\langle\ln A\rangle)$, because in the region $\sim(1-3) 10^{17} \mathrm{GeV}$ the dominance of heavy nuclei switches to dominance of protons in CR flux.

In Fig. 12 three energy dependencies of $\langle\ln A\rangle$ corresponding to the three spectra presented in Fig. 11 are shown. For the 
PROTON
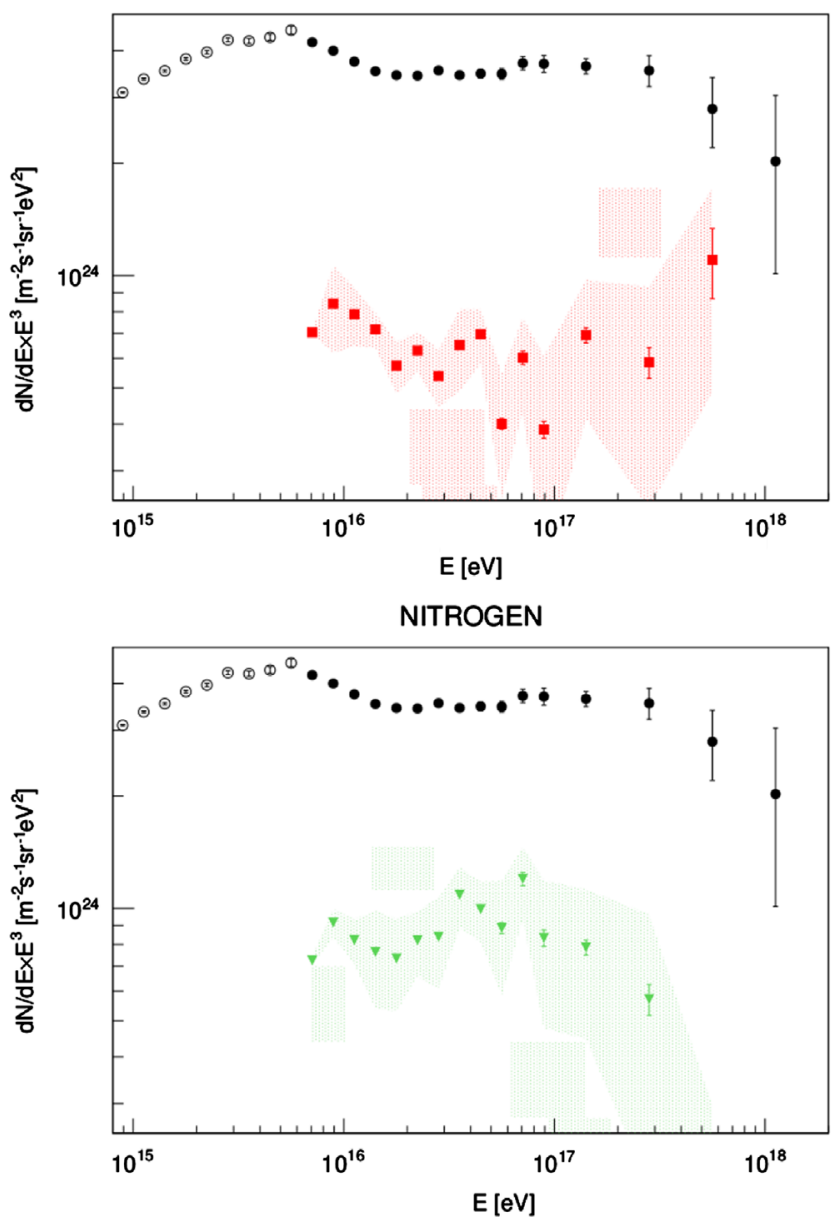

HELIUM
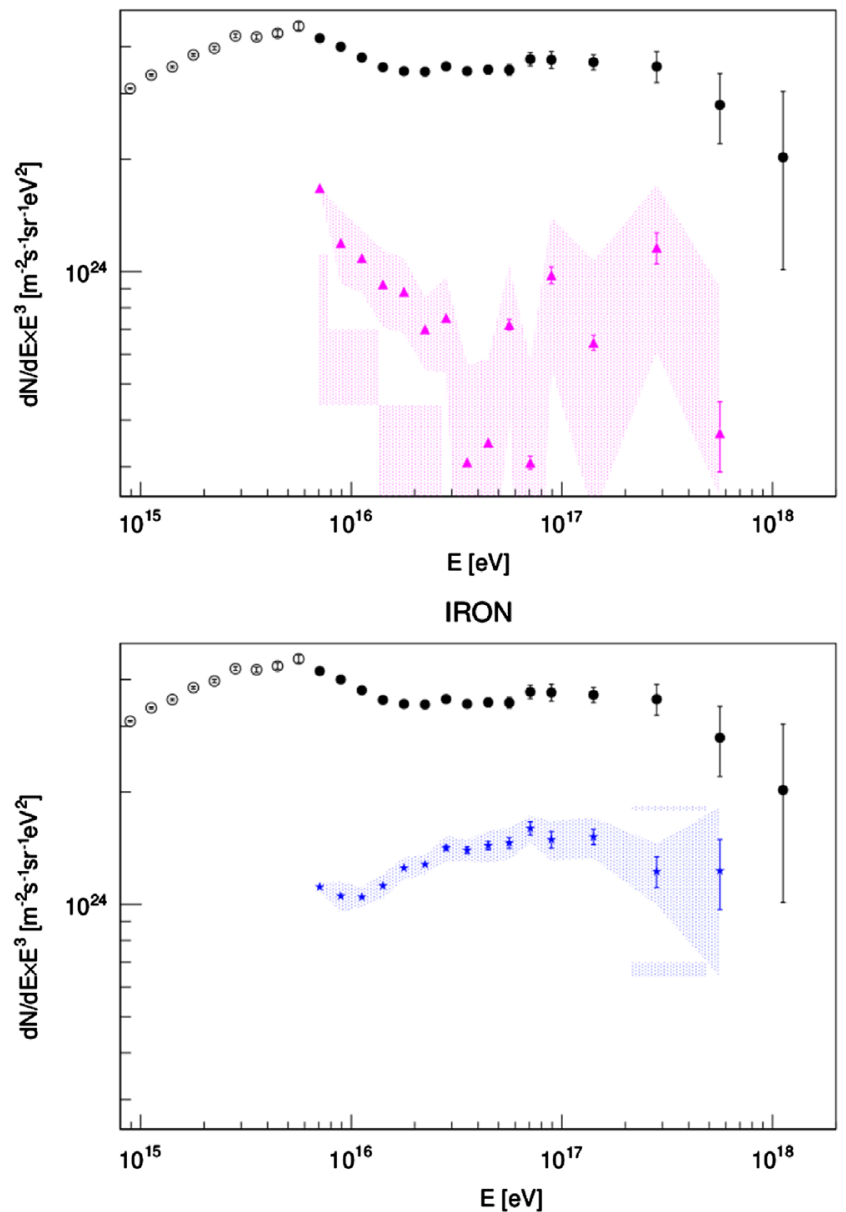

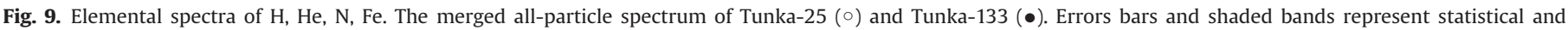
systematic uncertainties.

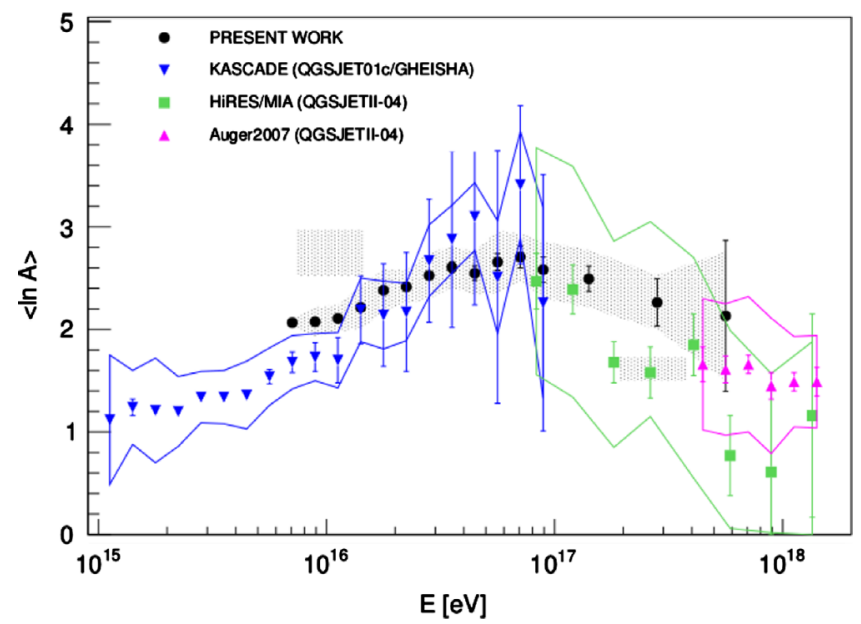

Fig. 10. $\langle\ln A\rangle$ as a function of energy. Errors bars and bands represent statistical and systematic uncertainties.

extragalactic component we assumed that $\langle\ln A\rangle=0.5$ corresponding to the composition $\sim 75 \%$ of protons and $\sim 25 \%$ of He nuclei. If we assume a pure proton composition, then $\langle\ln A\rangle=0$ at $E>10^{18} \mathrm{eV}$. The predicted maximum of $\langle\ln A\rangle$ value reaches $2.5-$ 3 , but in no case a value of 4 as in the KASCADE data [22]. In Fig. 12 we show the new Tunka data, and data from Ref. [1] presented earlier as well as some selective data from other experiments.
In the first approximation the third model fit (the black line) does not contradict to $\langle\ln A\rangle(E)$, measured in the Tunka 133 experiment [1], and the pink line fits new data. However, one can see that three last points in Fig. 11 and in Fig. 12 are slightly higher than calculated predictions. Is this difference only of statistical origin or there are some systematic errors in energy determination (stretched energy scale) is not clear yet. It is worth noting that it is impossible to move the position of a second knee to the right staying still in the frames of considered model.

To clarify this difference we show in Fig. 13 the predictions of our model (the same curves as in Fig. 11) for proton + He spectra in comparison with Tunka 133 'light' component (see Fig. 8). At Fig. 14 we show the model prediction for all nuclei with $Z>6$ and 'heavy component' (Fig. 8). At Fig. 14 some excess of heavy components is also seen in the region of $(2 \div 5) 10^{17} \mathrm{eV}$.

This excess in the region of $(2-5) \times 10^{17} \mathrm{eV}$ may be interpreted as a contribution of one galactic source (such as Cas A [16]), but cosmic rays produced by this source should have an abnormal chemical composition [16].

\section{Conclusions}

1. The primary spectrum above the knee cannot be fitted with a single power law index but with three indices: $3.23 \pm(0.01)^{\text {stat }}$ $\pm(0.05)^{\text {syst }}$ for $6 \times 10^{15}-2 \times 10^{16} \mathrm{eV}, 3.00 \pm(0.01)^{\text {stat }} \pm(0.05)^{\text {syst }}$ for $2 \times 10^{16}-3 \times 10^{17} \mathrm{eV}, 3.33 \pm(0.15)^{\text {stat }} \pm(0.05)^{\text {syst }}$ for $3 \times 10^{17}$ $-10^{18} \mathrm{eV}$. 


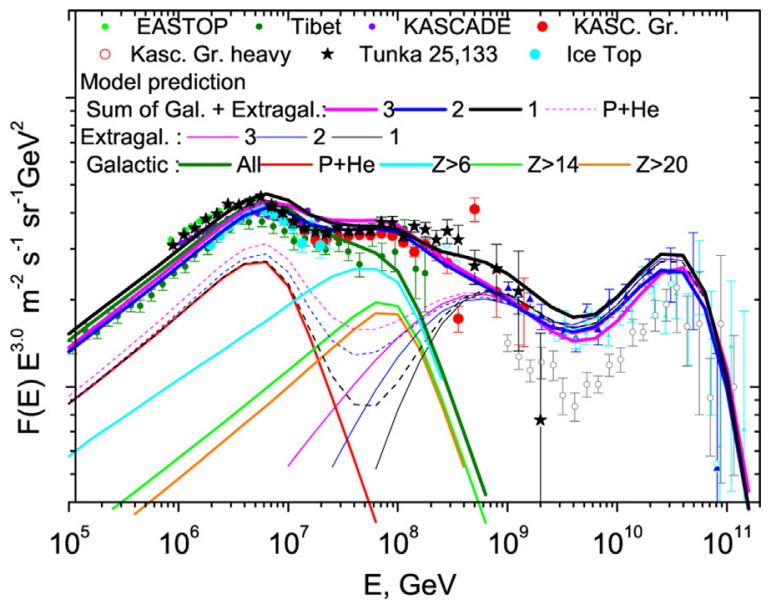

Fig. 11. All particle spectrum measured by different experiments: Tunka-133, Tunka 25 [19], KASCADE Grande [7], KASCADE [22], EASTOP [21], Tibet [20], UHE data, obtained in Hires 1 [23], Auger [24]. Galactic cosmic rays are presented separately: dark green line (total), red line $(\mathrm{H}+\mathrm{He})$, blue line $(Z>6)$, green one $(Z>14)$, orange one $(Z>20)$. Thin lines denote 3 fits of extragalactic protons, covered predictions [18]. Thick lines of the same colours represent galactic + extragalactic particles. Dashed lines denote the total light component: $\mathrm{p}+\mathrm{He}$. (For interpretation of the references to colour in this figure caption, the reader is referred to the web version of this paper.)

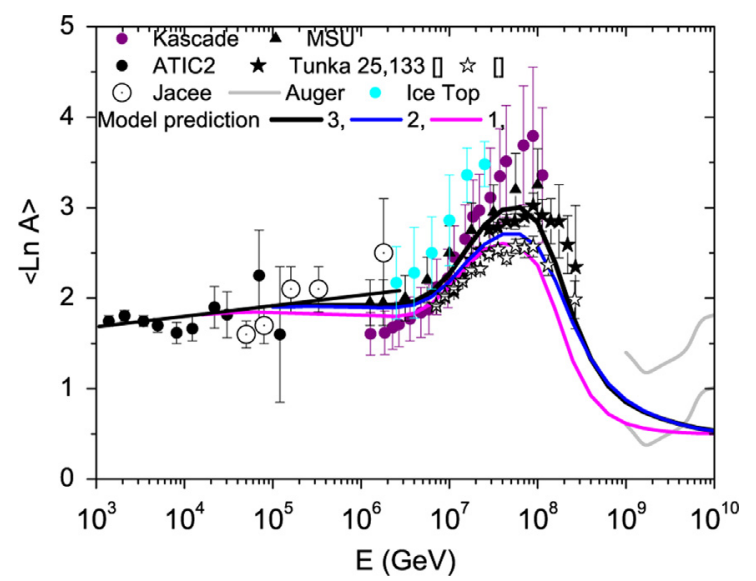

Fig. 12. $\langle\ln A(E)\rangle$ dependence corresponding to the 3 cases presented in Fig. 11 and selective experimental data: Atic2 [25], Jacee [26], KASCADE [27], Tunka-133 [1] (black sters) and unfilled stars (from Fig. 6), MSU [28], upper and lower limits for Auger's $\langle\ln A\rangle(E)$ dependence are taken from Ref. [29].

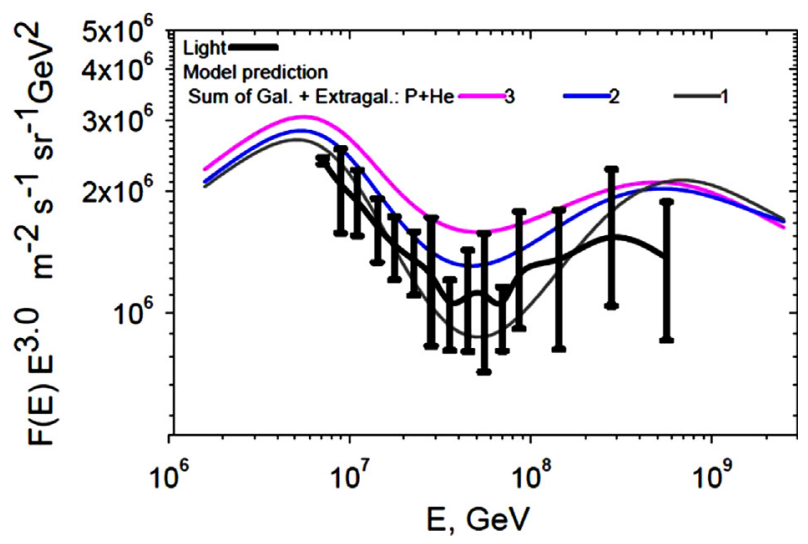

Fig. 13. Light component energy spectrum of Tunka 133 (with systematic errors) (Fig. 8) in comparison with the model predictions (lines are shown by the same colour as in Fig. 11). (For interpretation of the references to colour in this figure caption, the reader is referred to the web version of this paper.)

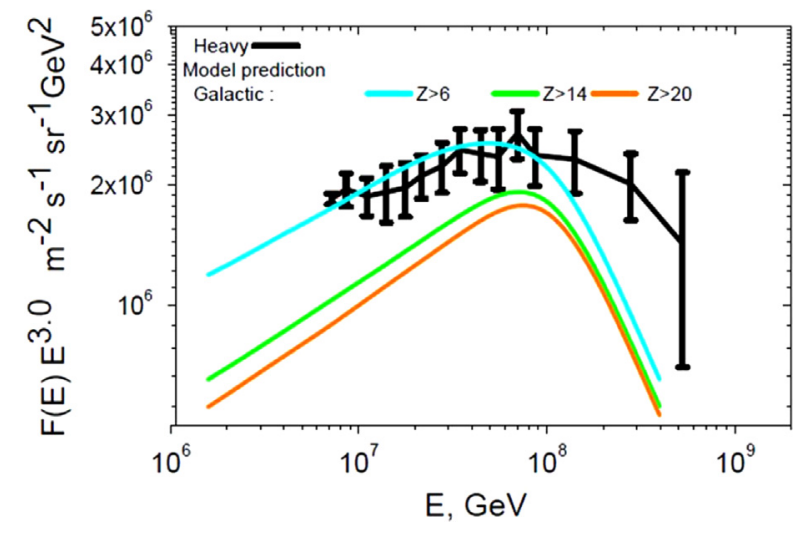

Fig. 14. Heavy component energy spectrum of Tunka 133 (Fig. 8) in comparison with the model predictions (lines are shown by the same colour as in Fig. 11). (For interpretation of the references to colour in this figure caption, the reader is referred to the web version of this paper.)

2. The high-energy tail of the spectrum is compatible with the Fly's Eye, HiRes and TA spectra.

3. The $X_{\max }$ values are compatible with that of HiRes and Auger.

4. The mass composition changes to a heavier composition in the energy range of $10^{16}-3 \times 10^{16} \mathrm{eV}$, stays heavy till $10^{17} \mathrm{eV}$ and starts changing to a lighter composition at energies higher than $10^{17} \mathrm{eV}$.

\section{Acknowledgments}

This work was supported by the Russian Federation Ministry of Education and Science (G/C 14.518.11.7046, 14.B25.31.0010, 14.14.B37.21.0785, 14.B37.21.1294), the Russian Foundation for Basic Research (Grants 11-02-00409, 13-02-00214, 13-02-12095, 13-02-10001)

\section{References}

[1] F. Berezhnev, et al., Tunka Collaboration, Nucl. Instrum. Methods A 692 (2012) 98.

[2] B. Antokhonov, et al., Tunka Collaboration, Nucl. Phys. B: Proc. Suppl. 212-213 (2011) 247.

[3] E.E. Korosteleva et al.n in: Proceedings of 31th ICRC, Lodz, Poland, vol. 1, 2009, p. 0492.

[4] E. Korosteleva, et al., Int. J. Mod. Phys. A 20 (2005) 6837 arxiv:astro-ph/ 0411216

[5] E.E. Korosteleva, et al., Nucl. Phys. B: Proc. Suppl. 165 (2007) 74.

[6] N.M. Budnev, et al., Tunka Collaboration, in: Proceedings of 29th ICRC, Pune, India, vol. 6, 2005, pp. 257-260, arxiv:astro-ph/0511215.

[7] W.D. Apel, J.C. Arteaga-Velazquez, et al., KASCADE Gr. Collaboration, Astropart Phys. 36 (2012) 183.

[8] S.N. Epimakhov et al., Tunka Collaboration, in: 33th ICRC, July 2013. ID =0326

[9] S. Ostapchenko, et al., Phys. Rev. D 83 (2011) 014018.

[10] W.D. Apel, et al., Astrophys. J. 31 (2009) 86.

[11] W.D. Apel, et al., KASCADE-Grande Collaboration, Phys. Rev. D 87 (2013) 081101(R).

[12] T. Abu-Zayad, et al., Phys. Rev. Lett. 84 (2000) 4276.

[13] M. Unger, et al., P. Auger Collaboration, ICRC2007, arxiv:0706.1495.

[14] L.G. Sveshnikova, O.N. Strelnikova, V.S. Ptuskin, arxiv:1301.2028.

[15] V.S. Ptuskin, V.N. Zirakashvili, E.S. Seo, Astrophys. J. 718 (2010) 31.

[16] L.G. Sveshnikova, E.E. Korosteleva, L.A. Kuzmichev, et al., J. Phys.: Conf. Ser. 409 (1) (2013) 012062 arxiv:1303.1713.

[17] V. Berezinsky, A.Z. Gazizov, S.I. Grigorieva, Phys. Lett. B 612 (2005) 147 arxiv: astro-ph/0502550.

[18] K. Kotera, M. Lemoine, Phys. Rev. D 77 (2008) 23005 arxiv:astro-ph/0706.1891.

[19] E.E. Korosteleva, et al., Tunka 25 Collaboration, Nucl. Phys. B: Proc. Suppl. 165 (2007) 74.

[20] M. Amenomori, X.J. Bi, D. Chen, et al., Tibet Collaboration, Astrophys. J. 678 (2008) 1165 arxiv:astro-ph/0801.1803.

[21] M. Aglietta, et al., EAS-TOP Collaboration, Astropart. Phys. 10 (1999) 1.

[22] T. Antoni, et al., KASCADE Collaboration, Astropart. Phys. 24 (2005) 1.

[23] R.U. Abbasi, et al., HiRes Collaboration, Phys. Rev. Lett. 100 (2008) 101101 arxiv:astro-ph/0703099. 
[24] J. Abraham, et al., Pierre Auger Collaboration, Phys. Rev. Lett. 101 (2008) 061101 arxiv:astro-ph/0806.4302.

[25] A.D. Panov, J.H. Adams Jr., H.S. Ahn, et al., ATIC2 Collaboration, Bull. Russ. Acad. Sci.: Phys. 73 (5) (2009) 564.

[26] Y. Takahashi, JACEE Collaboration, Nucl. Phys. B: Proc. Suppl. 60 (1998) 83.
[27] V. Roth, H. Ulrich, in: Proceedings of 28th ICRC, Japan, Tsukuba, vol. 1, 2003, p. 139.

[28] N.N. Kalmykov, G.V. Kulikov, V.P. Sulakov, et al., Moscow Univ. Phys. Bull. 64 (5) (2009) 536.

[29] P. Abreu, Auger Collaboration arxiv:1301.663. 